\title{
Original
}

\section{Nurses' Awareness of Cognitive Impairment in Individuals with Amyotrophic Lateral Sclerosis}

\author{
Mitsuko Ushikubo ${ }^{1}$, Tadahiro Ohtani ${ }^{2}$ and Hiromi Kawabata ${ }^{3}$ \\ 1 Gunma University Graduate School of Health Sciences, 3-39-22 Showa-machi, Maebashi, Gunma 371-8514, Japan \\ 2 Department of Nursing, Gunma University Hospital, 3-39-15 Showa-machi, Maebashi, Gunma 371-8511, Japan \\ 3 Nursing Department, Mihara Memorial Hospital, 366 Oota-machi, Isesaki, Gunma 372-0006, Japan
}

\begin{abstract}
Purpose: The number of individuals with amyotrophic lateral sclerosis (ALS) who also have cognitive impairment has increased. The purpose was to elucidate nurses' awareness of patients with comorbid ALS and cognitive impairment.

Methods: We conducted a survey of nurses with experience in supporting individuals with ALS, using an anonymous, self-administered questionnaire. The questionnaire collected information on the participant characteristics, awareness of the presence of cognitive impairment in ALS, their experience in supporting ALS patients with cognitive impairments, and their educational needs. We used descriptive statistics.

Results: We analyzed 117 questionnaires (response rate: 68.0\%). Seventy-one nurses (60.7\%) were aware of the cognitive impairments associated with ALS, and had only learned about this within the last one or two years, through their own clinical experience. 55 nurses $(43.6 \%)$ had current or past experience supporting patients with comorbid cognitive impairment and ALS. Approximately $20 \%$ of whom answered that never had cared for ALS patients diagnosed with cognitive impairment had experience of supporting an ALS patient suspected of having cognitive impairment. More than $80 \%$ of the respondents expressed their wishes to receive recurrent education regarding ALS patients with cognitive impairment.

Conclusions: The nurses in this study did not have a comprehensive understanding of cognitive impairment in ALS. Nurses need to be aware of the overlap in symptoms of ALS and cognitive impairment by receiving training to upgrade their knowledge.
\end{abstract}

\begin{tabular}{l} 
Article Information \\
\hline Key words: \\
amyotrophic lateral sclerosis, \\
cognitive impairment, \\
neurological nursing, \\
comorbidity \\
\hline Publication history: \\
Received: May 20, 2021 \\
Revised: June 17, 2021 \\
Accepted: June 30, 2021 \\
Corresponding author: \\
Mitsuko Ushikubo \\
Graduate School of Health Sciences, Gunma University, \\
3-39-22 Showa-machi, Maebashi, Gunma 371-8514, Japan \\
Tel: +81-27-220-8987 \\
E-mail: ushi2@gunma-u.ac.jp
\end{tabular}

\section{Introduction}

Amyotrophic lateral sclerosis (ALS) is a rapidly progressive and fatal neurodegenerative disorder for which there is no effective curative treatment available. ALS selectively affects the motor system and is clinically manifested as progressive decline in physical, swallowing, communication, and respiratory function. It significantly makes patients' activities of daily life toilsome. ${ }^{1}$

Although cognitive functions were thought to be spared, it is now known that a range of cognitive impairments can be involved in ALS. ${ }^{2}$ The prevalence of ALS with cognitive impairment varies in the literature. One study indicated that approximately $50 \%$ of patients with ALS have some type of cognitive dysfunction and $10-15 \%$ meet the diagnostic criteria for dementia. ${ }^{3}$ According to the investigation of 287 patients with sporadic ALS hospitalized at Gunma University Hospital, the percentage of ALS patients with dementia increased from $0 \%$ in the period of $1978-1982$ to $20.2 \%$ in 2008 $2012 .^{4}$

While there may be growing research into cognitive functioning in ALS, there is scarce research about the impact this has on caregivers or about how to provide best care for these patients..$^{5-7}$ In addition, no studies focused on nurses' awareness about comorbid ALS and cognitive impairment. The purpose of this study was to elucidate nurses' awareness of, and experiences with, 
patients with ALS and cognitive impairment in order to enhance the quality of care for these patients.

\section{Methods}

\section{Sample and data collection}

This was a cross-sectional, quantitative study. The participants were nurses with experience in supporting at least one ALS patient. Nurses were recruited from two hospitals, 38 visiting nursing agencies, and 11 public health centers in Prefecture A. We conducted an anonymous, self-administered questionnaire survey among 172 nurses. Questionnaires were distributed to hospital nurses by head nurses and the potential participants were asked to put the completed questionnaire in a collection box, while home health and public health nurses received and returned the questionnaire by mail.

We used part of the information collected by the questionnaire: the participants' characteristics, the nurses' awareness and experience, and willingness to participate in workshops regarding comorbidity of cognitive impairment and ALS. Nurses were asked whether they knew about the comorbidity of cognitive impairments with ALS. If they responded with an affirmative, they were asked when and how they learned about it. We also asked whether they had experience supporting ALS patients with cognitive impairment. If the answer to that question was not affirmative, we inquired about their experience with ALS patients suspected of having cognitive impairment.

\section{Data analysis}

Descriptive statistics (frequencies, mean, range, or percentage) were calculated for participant characteristics, nurses' awareness about the coexistence of cognitive impairment with ALS, and nursing experience supporting patients with comorbid ALS and cognitive impairment.

\section{Ethical considerations}

This study was conducted after obtaining approval from the Ethical Review Committee for Medical Research Involving Human Subjects of Gunma Univer- sity (No. 2018-040). This study was also approved by the ethical review committees of two hospitals. Participants were informed on the cover of the questionnaire about the purpose of the study, methods, and ethical considerations: confidentiality would be maintained, participation and withdrawal were entirely voluntary, and consent would be considered to have been granted with return of the questionnaire.

\section{Results}

A total of 121 responses were collected. Of these, three indicated no experience with ALS patients, and one response was illegible, and these were excluded from the study. Finally, we analyzed 117 questionnaires (response rate: $68.0 \%$ ).

As shown in Table 1, most participants in this study had many years of neurological nursing experience and had cared for numerous ALS patients. About half of the participants worked in a hospital, and the other half worked as home health nurses or public health nurses supporting patients with intractable neurological diseases.

As shown in Table 2, 60\% of participants were aware of the presence of cognitive impairments in ALS. Many of them had only learned about this within the last 1 or 2 years, often through their own clinical experience.

As shown in Table 3, nearly half of the participants had current or past experience supporting patients with comorbid cognitive impairment and ALS, and although several participants had never cared for ALS patients diagnosed with cognitive impairment, nearly 20\% had experience in supporting an ALS patient suspected of having cognitive impairment.

The willingness to participate in workshops on ALS with cognitive impairment was shown in Table 4. More than $80 \%$ of the respondents wished to participate in the workshop, including "strongly desire to participate" and "may participate". In terms of workplaces, home visiting nurses and public health nurses tended to have a high desire to participate.

Table 1 Participant characteristics

$(\mathrm{n}=117)$

\begin{tabular}{lcc}
\hline Clinal experience & Mean & Range \\
\hline Nursing experience & 15.9 years & 6 months-40 years \\
Neurological nursing experience & 6.2 years & 6 months-24 years \\
\hline Number of patients with ALS whom participants had supported & $\mathrm{n}$ & $\%$ \\
\hline 1-5 patients & 36 & 30.8 \\
6-10 patients & 20 & 17.1 \\
Over 11 patients & 59 & 2 \\
No response & 50.4 & 1.7 \\
\hline Workplaces & $\mathrm{n}$ & $\%$ \\
\hline Hospitals & 62 & 53 \\
Home visiting nursing stations & 41 & 35 \\
Prefectural office/Public health centers & 14 & 12 \\
\hline
\end{tabular}

ALS: amyotrophic lateral sclerosis. 
Table 2 Nurses' awareness of the comorbidity of cognitive impairment in ALS

\begin{tabular}{|c|c|c|}
\hline Do you know about the comorbidity of cognitive impairment with ALS? & $\mathrm{n}$ & $\%$ \\
\hline Yes & 71 & 60.7 \\
\hline No & 46 & 39.3 \\
\hline Among the participants who answered yes & \multicolumn{2}{|c|}{$\mathrm{n}=71$} \\
\hline$<$ When did you learn about this? $>$ & $\mathrm{n}$ & $\%$ \\
\hline Within the last $1-2$ years & 41 & 57.7 \\
\hline Within the last 3-5 years, or more & 24 & 33.8 \\
\hline No response & 6 & 8.5 \\
\hline$<$ How did you learn about this? $>$ multiple answers possible & $\mathrm{n}$ & $\%$ \\
\hline From clinical experience & 54 & 76 \\
\hline From books or journals & 13 & 18.3 \\
\hline From nursing colleagues & 11 & 15.5 \\
\hline From physicians & 14 & 19.7 \\
\hline From professional conferences or seminars & 18 & 25.4 \\
\hline From TV programs & 2 & 2.8 \\
\hline Others & 1 & 1.4 \\
\hline
\end{tabular}

Table 3 Nursing experience supporting ALS patients with comorbid cognitive impairment

Do you have any experience providing support to ALS patients with cognitive impairment?

Yes, I am currently supporting these type of patients

$\mathrm{n}=117)$

Yes, I have previous experience, but I do not do it anymore

$\%$

Never

Unknown

9.4

34.2

No response

38.5

17.1

Among responses of either 'never' or 'unknown'

0.9

Do you have any experience supporting ALS patients who needed cognitive tests or medical examinations? $\quad$ n

Yes, I am currently supporting a patient of that type

Yes, I have previous experience, but I am not providing support to these patients currently

Unknown

No response

Table 4 Willingness to participate in workshop by workplaces

n $(\%) \quad(n=117)$

\begin{tabular}{|c|c|c|c|c|c|}
\hline $\begin{array}{l}\text { Willingness to } \\
\text { participate in workshop }\end{array}$ & Workplace & $\begin{array}{l}\text { Hospitals } \\
\qquad n=62\end{array}$ & $\begin{array}{l}\text { Home visiting } \\
\text { nursing stations } \\
\qquad \mathrm{n}=41\end{array}$ & $\begin{array}{l}\text { Prefectural office or } \\
\text { health care centers } \\
\qquad n=14\end{array}$ & total \\
\hline Strongly desire & & $18(29.0)$ & $16(39.0)$ & $8(57.1)$ & $42(35.9)$ \\
\hline May participate & & $31(50.0)$ & $21(51.2)$ & $4(28.6)$ & $56(47.9)$ \\
\hline Will not participate & & $12(19.3)$ & $4(9.8)$ & $2(14.3)$ & $18(15.4)$ \\
\hline No answer & & $1(1.6)$ & $0(0.0)$ & $0(0.0)$ & $1(0.9)$ \\
\hline
\end{tabular}

\section{Discussion}

This study explored the nurses' awareness of cognitive problems in ALS and their experiences supporting such patients. Cognitive and behavioral symptoms in ALS have been described for over a century. It appears that the nurses in this study did not have a comprehensive understanding of cognitive impairment in ALS, given that the majority of them had been caring for ALS patients for many years, yet had only learned about the co-occurrence of the two conditions in the last year or two. One study suggested that neurologists in clinical settings should pay more attention to the cognitive and behavioral aspects of patients with ALS. ${ }^{8}$ Nurses working in various care facilities should also recognize comorbid ALS and cognitive impairment by receiving training to upgrade their knowledge.

Among those who answered that they did not have experience supporting individuals with ALS and cognitive impairment, or who were unsure, about $20 \%$ had experience supporting ALS patients who they suspected had cognitive impairments and needed cognitive testing. In other words, it is presumed that the necessary examinations were not performed even though the nurses suspected that the patients had cognitive impairment. Motor deficits caused by ALS may interfere with the performance of cognitive tests. For instance, hand weakness, dysarthria, or a lack of verbal communication abilities can make it impossible for patients to respond to cognitive tests in written or oral format. Furthermore, in-home patients with ALS with severe disabilities have difficulties moving, and so they are unable, or do not want, to travel to hospitals for magnetic resonance imaging (MRI) or other procedures.

In this study, hospital nurses, home health nurses, and public health nurses were targeted in order to gather 
nurses' awareness and experience in various settings. More than $80 \%$ of them wanted to participate in the workshop on ALS with cognitive impairment. More home health nurses and public health nurses than hospital nurses wanted to participate in the workshop, suggesting that these nurses cared for the patients for a long period of time. Educational opportunities should be provided to meet their learning needs, and to upgrade their knowledge. Thereby, nurses who provide care for ALS patients in various settings will be possible to pay more attention to cognitive impairment and develop care strategies that integrate the knowledge and care skills of cognitive impairment into ALS care.

It is difficult to generalize the results of this study due to the limited number of participants from one area. Further studies should include larger sample sizes. If possible, participants should be recruited from other locations. We also recommend conducting interviews to collect more detailed information about nurses' experiences.

\section{Conclusion}

This study clarified the level of nurses' awareness and experiences in supporting patients with ALS and cognitive impairment. The nurses in this study did not have a comprehensive understanding of cognitive impairment in ALS. It was presumed that there were situations in which it was difficult to perform appropriate clinical examinations for in-home ALS patients, even if cognitive impairment was suspected.

\section{Acknowledgments}

We thank the nurses for collaborating in this study. This work was supported by JSPS KAKENHI (grant number 18K10304).

\section{Declarations of interest}

The authors declared no potential conflicts of interest.

\section{References}

1. Hobson EV, McDermott CJ. Supportive and symptomatic management of amyotrophic lateral sclerosis. Nat Rev Neurol 2016; 12: 526-538.

2. Phukan J, Pender NP, Hardiman O. Cognitive impairment in amyotrophic lateral sclerosis. Lancet Neurol 2007; 6: 9941003.

3. Lomen-Hoerth C, Murphy J, Langmore S, et al. Are amyotrophic lateral sclerosis patients cognitively normal? Neurology 2003; 60; 1094-1097.

4. Furuta N, Makioka K, Fujita Y, et al. Changes in the clinical features of amyotrophic lateral sclerosis in rural Japan. Intern Med 2013;52:1691-1696. doi:10.2169/internalmedicine. 52. 0195.

5. Caga J, Hsieh S, Lillo P, et al. The impact of cognitive and behavioral symptoms on ALS patients and their caregivers. Front Neurol 2019; 10: 192.

6. Cui B, Cui L, Liu M, et al. Behavioral symptoms in motor neuron disease and their negative impact on caregiver burden. Chin Med J 2015; 128: 2295-2300.

7. Lillo P, Mioshi E, Hodges JR. Caregiver burden in amyotrophic lateral sclerosis is more dependent on patients' behavioral changes than physical disability: A comparative study. BMC Neurol 2012; 12: 156.

8. Watanabe Y, Raaphorst J, Izumi, Y, et al. Cognitive and behavioral status in Japanese ALS patients: a multicenter study. J Neuro 2020; 267: 1321-1330. 